\title{
SOSTENIBILIDAD FISCAL EN COSTA RICA, 1991- 2013: UNA APROXIMACIÓN A TRAVÉS DEL MÉTODO MONTECARLO
}

\section{FISCAL SUSTAINABILITY IN COSTA RICA 1991-2013: A MONTECARLO APPROACH.}

\author{
Julio César Espinosa Rodríguez ${ }^{1}$ \\ Marco Vinicio Valerio Berrocal ${ }^{2}$
}

Toda la diferencia entre un mal y un buen economista es esta: uno se limita al efecto visible; el otro tiene en cuenta el efecto que se ve y los

que hay que prever

Frédéric Bastiat, 1848

\begin{abstract}
Resumen
El presente documento es una aplicación de un modelo de sostenibilidad fiscal para la economía de Costa Rica. Se utiliza la metodología de límite natural de deuda propuesta por Mendoza y Oviedo (2009) y Simulaciones Montecarlo para calcular la probabilidad de superar este límite. El modelo presentado es una simplificación del aplicado por Tanner y Samake (2007) y Fernández (2005). Al pronosticar 3 años se obtiene un 76,09\% de probabilidad de superar el límite de deuda definido como crítico, lo que indica que la economía posee un alto riesgo de insostenibilidad de la deuda. Es por ello que basado en funciones de impulsorespuesta se concluye el posible resultado de una política fiscal contractiva.
\end{abstract}

Palabras clave: Simulación; Método Montecarlo; sostenibilidad fiscal.

\footnotetext{
Fecha de recibido: 19 de mayo del 2014, Fecha de reenvíos: 20 de junio del 2014, 24 de junio del 2014, Fecha de aceptación: 28 de junio del 2014, Fecha de publicación: 30 de junio del 2014

doi: http://dx.doi.org/10.15359/eys.19-45.3

${ }_{1}^{1}$ Máster en Economía, docente e investigador de la Escuela de Economía, Universidad Nacional, Costa Rica, crinvestments@racsa.co.cr

${ }^{2}$ Bachiller en Economía, Estudiante Asistente de la carrera de Economía, Universidad Nacional, Costa Rica, markxsw@gmail.com
} 


\begin{abstract}
The following document is an application of the fiscal sustainability model for Costa Rican economy. The model uses the methodology of natural debt limit proposed by Mendoza and Oviedo (2009) as well as a set of Montecarlo simulations to estimate the probability of surpassing this limit. The model is a simplification of the one applied by Tanner and Samake (2007) and Fernandez K. (2005). After forecasting a 3 year period a $76.09 \%$ probability to exceed the limit of debt was obtained indicating that the economy has a high risk of unsustainable debt, in this context is was proposed, based on the IRF's, a possible contractive fiscal politic was encountered.
\end{abstract}

Keywords: Simulation; Montecarlo method; Fiscal sustainability.

\title{
Introducción
}

El manejo de la política fiscal es fundamental para cualquier país, debido al impacto que esta puede tener sobre el consumo, la inversión y la producción. La literatura muestra que incrementos del gasto público y reducciones de los impuestos causan variaciones transitorias en el consumo e inversión, que tienen efectos positivos sobre la demanda agregada, estabilizan la economía en etapas recesivas del ciclo económico, e incurren generalmente en un déficit fiscal. Por otro lado, la política fiscal puede causar impactos negativos sobre la economía cuando el déficit fiscal es crónico, esto provocará aumentos en la deuda pública que, de no ser administrados, presionarán el alza de las tasas de interés, de tal manera que reducen el consumo e inversión, por lo tanto, la demanda agregada a mediano y largo plazo. Es en este contexto donde la sostenibilidad de la deuda cobra importancia, ya que es necesario implementar una política fiscal que no comprometa la estabilidad de la economía.

Tanner y Samake (2007) definen que una política fiscal sostenible es aquella que puede continuarse en el futuro sin ser modificada. En países como Costa Rica que se caracterizan por déficits fiscales recurrentes, la sostenibilidad de la deuda debe de ser un tema de preocupación para los hacedores de política económica, ya que una deuda insostenible coloca al país en una posición de vulnerabilidad.

Para valorar la sostenibilidad fiscal existen dos enfoques el ex post y el ex ante. El primero, ex post, que examina el cumplimiento de la restricción intertemporal del gobierno mediante una relación de largo plazo entre los ingresos y los gastos del sector público. En este enfoque se encuentran varios indicadores, por ejemplo: la Brecha FiscalCrecimiento Económico, basada en el trabajo de Acevedo (2001), la brecha tributaria de corto y mediano plazo de Blanchard (1990) y el indicador de sostenibilidad fiscal de Talvi y Vegh (1998), todos estos se interpretan como una magnitud, ya sea de crecimiento (Indicador de Acevedo) o de déficit fiscal como porcentaje al producto interno bruto (PIB) (Indicadores de Blanchard y Talvi Vegh), necesaria para mantener estable la relación entre la deuda pública y el PIB. En el segundo enfoque, ex ante, que se fundamenta en el análisis de la estimación futura de los ingresos, los gastos, el endeudamiento y otras variables 
que dependen del modelo utilizado. Dentro de este se encuentran diversos modelos que son utilizados para generar pronósticos de los flujos de ingresos y gastos del gobierno mediante diversos escenarios.

Cabe resaltar que ambos enfoques no son excluyentes, sino complementarios. La principal ventaja de los métodos ex post es que se alimenta de las variables históricas lo que propicia un análisis descriptivo de la situación fiscal, por lo que funciona como punto de referencia para el diseño de políticas fiscales. Como desventaja se encuentra el hecho de que al utilizar información histórica brinda información regazada a los diseñadores de la política fiscal, además las recomendaciones realizadas mediante este enfoque no admite la posibilidad de realizar experimentos contrafactuales.

Por otra parte, los modelos ex ante tienen la capacidad de generar diversos experimentos contrafactuales que brindan un insumo importante para el diseño de la política fiscal, ya que permiten valorar distintos escenarios, pero su principal desventaja corresponde a la validez de los supuestos realizados para generar los pronósticos, puesto que estos no siempre se cumplen, además bajo este enfoque se trabaja con incertidumbre, los pronósticos son un rango y las estimaciones puntuales de los mismos no siempre son acertadas.

Precisamente lo elevado que ha llegado a ser el servicio de la deuda para el presupuesto de algunos países ha producido que la deuda pública se trasforme en un problema estructural muy grave, tanto para los países subdesarrollados como para los desarrollados. Por ejemplo Papadopoulo et al. (1999) demuestra que Bélgica, España, Italia, Grecia y Portugal, países de en la Unión Europea, presentan evidencia de insostenibilidad y se encuentran en riesgo de incumplir el tratado de Maastricht, el estudio se anticipa al problema de insostenibilidad que se manifestó en el 2010 durante la crisis griega. Además los problemas causados por una deuda insostenible se evidenciaron durante la crisis que tuvo lugar en los primeros años de la década de los ochentas en América Latina y que ameritó la creación de reformas estructurales.

Según lo anterior se ha hecho cada vez más urgente contar con indicadores que permitan medir la capacidad de los países para hacer frente a los niveles de deuda cada vez más altos, lo cual ha incentivado en las últimas décadas la construcción y utilización de indicadores de sostenibilidad fiscal.

Por otra parte, a pesar de que los hacedores de la política fiscal tengan las mejores intenciones de tener deudas sostenibles y déficits fiscales bajos, la evolución de otras variables como el tipo de interés, el tipo de cambio o el crecimiento de la economía pueden comprometer esas intenciones.

El presente documento desarrolla la medición de la sostenibilidad de la deuda de forma estocástica, utilizando el Método Montecarlo para calcular la probabilidad de superar un ratio deuda al PIB definido como crítico. El modelo es probabilístico, por lo tanto no pretende generar pronósticos de niveles de deuda. Este documento se organiza de la siguiente forma: en la segunda sección se presentan 
algunas consideraciones teóricas, mientras que en la tercera sección se explican los aspectos metodológicos y de manejo de datos para la realización de las simulaciones. Las secciones cuarta y quinta del documento presentan, respectivamente, una síntesis de la situación fiscal de Costa Rica en los últimos años y se muestran los principales resultados obtenidos a través de las simulaciones y del análisis de funciones de impulso-respuesta. Finalmente, en la sexta sección se exponen las conclusiones del análisis desarrollado.

\section{Consideraciones teóricas}

Keynes (1971) para el caso de Francia en los años 20 planteaba la necesidad de implementar políticas fiscales sostenibles, y de no lograrse la insostenibilidad se iba a manifestar en el momento en el que los pasivos llegaran a ser un porcentaje elevado del ingreso nacional, lo cual claramente obligaría al gobierno a elevar los impuestos y/o reducir los gastos.

En esta línea de análisis se puede decir que una política fiscal es sostenible, si el gobierno es capaz de hacer frente a sus obligaciones pactadas. De este modo esa sostenibilidad dependerá de los ingresos y gastos futuros del fisco, incluidos los intereses. De tal manera que, el gobierno no debería elevar su deuda a volúmenes cuyo pago de intereses ponga en peligro el balance fiscal, y por otro lado, que la deuda llegue a niveles elevados con respecto al PIB.

Con base en los estudios de Buiter $(\underline{1985})$ y Blanchard $(\underline{1990})$ han surgido diferentes propuestas de indicadores para evaluar la sostenibilidad fiscal, los cuales ayudan a tomar decisiones de política económica.

Estos indicadores miden si las políticas fiscales vigentes pueden o no estabilizar la razón activos netos al PIB (según Buiter) o la razón deuda al PIB (según Blanchard). Debido a que no es fácil conseguir información fiable sobre los activos netos del sector público, muchos prefieren utilizar la razón deuda al PIB. Para ello se realiza una proyección del ingreso y el gasto público con base en las políticas vigentes y se calculan los indicadores, luego con los déficits primarios y el coeficiente tributario proyectados se compara el déficit primario permanente o el coeficiente tributario permanente necesario para mantener constante la razón deuda PIB.

Según lo anterior, la sostenibilidad fiscal debe analizarse desde el contexto de la restricción presupuestaria intertemporal del sector público ${ }^{3}$ y las necesidades de financiamiento del sector público, las cuales se representan de la siguiente forma:

3 Con el fin de simplificar el análisis no se consideran temas tales como posibles ventas de activos públicos, el señoreaje y las revaluaciones de activos y pasivos, las que se consideran iguales a cero 
(1)

$$
N F_{t}=\left(D_{t}-D_{t-1}\right)=D p_{t}+r_{t} D_{t-1}
$$

$\mathrm{NF}_{\mathrm{T}}: \quad$ necesidad de financiamiento del sector público en el periodo $t$

$\mathrm{Dp}_{\mathrm{t}}$ : déficit primario en el periodo $t$

$r_{t} D_{t-1}$ : pagos de intereses correspondientes a la deuda pública

$\mathrm{D}_{\mathrm{t}}-\mathrm{D}_{\mathrm{t}-\mathrm{i}}$ : cambio en el saldo de deuda total

De ese modo la necesidad de financiamiento en el periodo t lleva a un cambio en el saldo de la deuda total para financiar el déficit primario y los pagos por intereses de la deuda pública.

Al despejar Dpt se tiene:

$$
D p_{t}=r_{t} D_{t-1}-\left(D_{t}-D_{t-1}\right)
$$

Por medio de esta relación se deriva la condición básica de sostenibilidad, la cual establece una relación entre el crecimiento de la deuda, del PIB y el déficit primario, dada determinada tasa de interés.

Ahora bien, la ecuación (2) se puede dividir por el PIB, y asumir que la economía crece a una tasa exógena $y$ a largo plazo por lo que se obtiene:

$$
d_{t}=\left(1+r_{t}-\gamma_{t}\right) d_{t+1}-\left(t_{t}-g_{t}\right)
$$

Donde:

$d_{t}:$ ratio de deuda madura como porcentaje del producto en $t$

$d_{t+1}$ : ratio de la deuda pública emitida en $t$ con fecha de maduración $\mathrm{t}+1$ como porcentaje del PIB nominal

$r_{t}: \quad$ tasa de interés real de la deuda pública

$\gamma_{t}: \quad$ tasa de crecimiento del PIB a largo plazo

t: $\quad$ ratio de ingreso público al PIB nominal

gt: ratio del gasto público, se excluye pago de intereses, al PIB nominal

Esta ecuación nos permite analizar la relación deuda/PIB de cada nuevo periodo, la cual será el resultado de la deuda emitida, su tasa de interés, el crecimiento de la economía y el resultado primario del sector público global.

\section{Modelo propuesto}

El modelo propuesto es un modelo de equilibrio parcial basado en la restricción presupuestaria del gobierno, que tiene como fin evaluar probabilísticamente la posibilidad de que la razón deuda/PIB supere un límite determinado como crítico.

La metodología que se utilizará consiste en generar trayectorias hipotéticas del ratio deuda/PIB; posteriormente, mediante el Método Montecarlo, se calcula la probabilidad de sobrepasar el límite crítico que define la sostenibilidad o no sostenibilidad de la deuda 
a largo plazo. El Método Montecarlo consiste en un proceso iterativo que calcula la probabilidad de que suceda un evento; en este caso el evento es superar este límite crítico de deuda. La probabilidad Montecarlo se encuentra representada por la ecuación 4.

$$
P\left(d_{t}>d^{*}\right)=\frac{\text { Númerode iteraciones que en un periodot ocurre un eventoX }}{\text { Total de iteraciones }}
$$

Para realizar las simulaciones es importante definir el comportamiento del stock de deuda, el cual es definido por la ecuación 3 presentada en el apartado teórico y que hace referencia a una ecuación en diferencia que modela el movimiento del stock de deuda. $\mathrm{A}$ esta ecuación se le llamará la "función de acumulación de deuda".

La función de acumulación de deuda depende de la tasa de interés, el crecimiento y el superávit primario. Su respuesta a las variables del sistema es la siguiente: cuando la tasa de interés crece los pagos de la deuda aumentan, por lo tanto eleva también el stock de deuda; el crecimiento de la economía en cambio ayuda a pagar la deuda, los niveles mayores de actividad económica aumentan la capacidad del país para enfrentar el pago de los pasivos. Con respecto al resultado primario del sector público global el efecto de un incremento de este obliga al Estado a cubrir esta diferencia entre ingresos y gastos con endeudamiento, de tal manera que se acumula más deuda.

Por otra parte, el margen critico de deuda $d^{\wedge *}$, es definido por Mendoza y Oviedo (2009) de la siguiente manera:

$$
d^{*}=\frac{\left|t^{m ! n}-g^{m ! n}\right|}{|r-\gamma|}
$$

\section{Donde: \\ $t^{\min }: \quad$ tasa de ingresos fiscales mínimos con una confianza del $95 \%$. \\ $g^{\min }: \quad$ tasa de gastos fiscales mínimos con una confianza del $95 \%$. \\ $r: \quad$ tasa interés implícita promedio. \\ $\gamma: \quad$ tasa de crecimiento promedio en estado estacionario.}

Este límite representa la proporción de deuda/PIB del peor escenario de ingresos fiscales, dado el comportamiento promedio del crecimiento de la producción y la tasa de interés. Este límite de deuda "permite que el gobierno ofrezca a sus acreedores un compromiso creíble para permanecer capaz de pagar "casi con seguridad" en todo momento (incluso durante las crisis fiscales). Es importante señalar que este compromiso no es una suposición ad hoc, implica distintos supuestos, por ejemplo: (a) el gobierno es reacio a sufrir un colapso de sus desembolsos, (b) los ingresos públicos son estocásticos y (c) los mercados de créditos contingentes son incompletos. Sin embargo, el compromiso es en términos de un "criterio de capacidad de pago", y como tal, no descarta escenarios de incumplimiento que puedan resultar de la "voluntad de pago" o por razones estratégicas"(Mendoza $y$ Oviedo, 2009).

\footnotetext{
${ }^{4} \mathrm{t}^{\mathrm{min}}$ Se define como el promedio incondicional de $t$ menos 1,96 veces el desvío estándar, análogamente $\mathrm{g}^{\mathrm{min}}$ se define de la misma manera. Como proxy de la tasa de crecimiento en estado estacionario se utiliza la tendencia de Hodrick y Prescott del crecimiento.
} 
La simulación de las trayectorias de deuda se realiza de la siguiente manera:

(6)

$$
d_{t}=\left(1+r_{t}(\operatorname{sim})-\gamma_{t}(\operatorname{sim})\right) d_{t-1}-f_{t}(\operatorname{sim})
$$

Donde:

$d_{t}: \quad$ proporción de deuda del periodo $t$.

$d_{t-1}$ : proporción de deuda del periodo anterior.

$f_{t}(\mathrm{sim})$ : $\quad$ superávit primario promedio más un shock aleatorio.

$r_{i}(\operatorname{sim}): \quad$ tasa de interés promedio más un shock aleatorio.

$\gamma_{t}(\mathrm{sim})$ : crecimiento en estado estacionario promedio más un shock aleatorio.

La propagación de los shocks aleatorios de las variables simuladas es modelada a través de un vector autorregresivo (VAR) con la siguiente especificación:

$$
w_{t}=\beta_{0}+\sum_{i=1}^{k} \beta_{i} w_{t-i}+\varepsilon_{t}
$$

Donde $w_{t}$ son las variables endógenas o de política económica, las variables incluidas son representadas por el vector definido a continuación:

$$
w_{t}=\left(\begin{array}{c}
\gamma_{t} \\
f_{t} \\
q_{t} \\
r_{t}
\end{array}\right) \quad \varepsilon_{t} \sim N(0,1)
$$

Las variables contenidas por el vector $\mathrm{w}_{t}$ son el crecimiento económico, la razón déficit fiscal al PIB, el tipo de cambio real y por último la tasa de interés.

$$
\varepsilon_{t}=M v_{t}
$$

Una vez estimado el modelo VAR es posible realizar las simulaciones de las variables endógenas, utilizando la descomposición de Cholesky para propagar los shocks aleatorios, como se mencionó anteriormente.

\section{Donde:}

W: $\quad$ Descomposición de Cholesky de la matriz $\Sigma$, tal que $\Sigma=\mathrm{MM}^{\prime}$

vt: $\quad$ Vector de shocks aleatorios distribuidos de manera idéntica a partir de una distribución estándar vt $\sim(N(0,1))$

Al utilizar la factorización de Cholesky definir el orden de las variables en el sistema resulta fundamental, ya que determina la dinámica del sistema. 
Por último, el criterio de decisión sobre la sostenibilidad de la deuda es el siguiente:

$d_{t} \geq d^{*}: \quad$ la deuda es insostenible.

$d_{t}<d^{*}: \quad$ la deuda es sostenible.

\section{Los datos}

Los datos de deuda, PIB, ingresos fiscales, gastos fiscales y crecimiento, que fueron utilizados son publicados por el Banco Central de Costa Rica (BCCR), con periodicidad trimestral. La estimación del Vector Autorregresivo se realiza con datos del periodo 1991 - 2012 con en el Software Eviews 7. La estimación de las funciones de impulsorespuesta es realizada mediante el método de impulsos ortogonales de Cholesky. Por otra parte, la estimación de probabilidades y las simulaciones de las trayectorias de deuda son realizadas en el Software Matlab R2010a, en donde se utilizan como valores iniciales las magnitudes de déficit, tasa de interés y deuda observados en el cuarto trimestre del 2013 y los valores de crecimiento económico pronosticados por la revisión del programa macroeconómico del Banco Central publicada en el tercer trimestre del 2013.

La simulación inicia en el primer trimestre del 2014 y finaliza en el tercer trimestre del 2006, y no considera escenarios. Además, la simulación utiliza 5000 iteraciones, esto para lograr convergencia en las probabilidades estimadas y obtener una simulación robusta.

Las series no son tratadas con filtros de estacionalidad, excepto el proxy de crecimiento ya mencionado, debido a que se puede eliminar información importante para la estimación. Esto concuerda con la posición de Hansen y Sargent (1993) que defiende el hecho de que los agentes toman decisiones sobre datos sin desestacionalizar, por lo tanto remover la estacionalidad implica una pérdida de información importante, además de que sesgan las estimaciones de los parámetros, como los del VAR.

Por otra parte, en estudios como el de Ghysels (1990) y luego Ghysels y Perron (1993) se ha determinado que los filtros de estacionalidad, como el X-11, agregan un sesgo positivo a las estimaciones de coeficientes asociados a un término autorregresivo y reducen la potencia de las pruebas de raíz unitaria.

\section{Situación fiscal de Costa Rica en los últimos años}

En este apartado se estimarán algunos estadísticos descriptivos con el fin de conocer diferentes hechos estilizados que caracterizan la política fiscal costarricense. En primer lugar se analizará la serie histórica del déficit fiscal a partir de 1991, para identificar las causas de distintos quiebres en la serie. Después de esto se examinará el comportamiento histórico de la deuda pública, tanto interna como externa para finalizar con un balance de riesgos basado en la ley del presupuesto nacional 2014. 


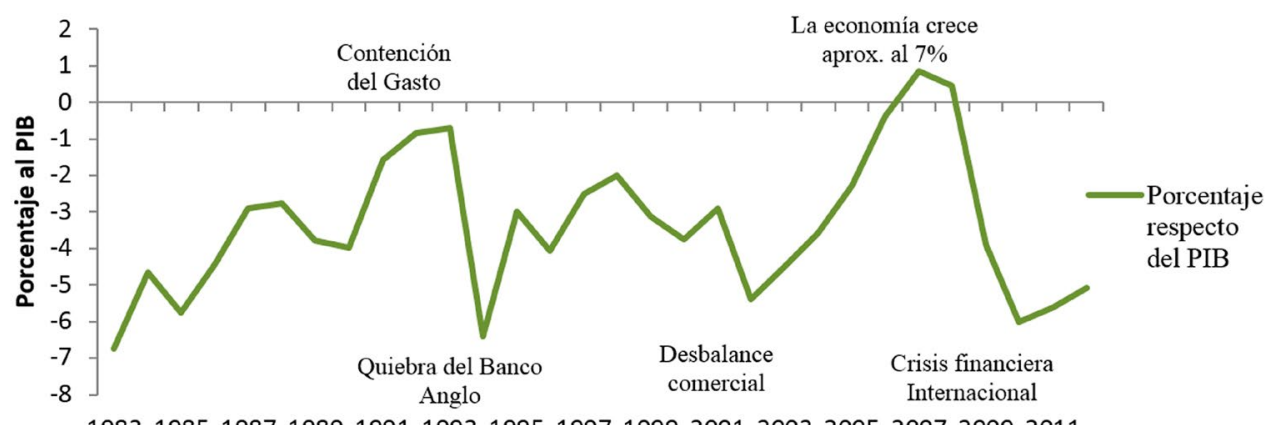

198319851987198919911993199519971999200120032005200720092011

Año

Figura 1. Resultado financiero del sector público global (1983-2012) -Porcentaje respecto del Producto Interno Bruto- Fuente: elaboración propia con base en datos del BCCR.

Los hechos más relevantes en la evolución del déficit fiscal son detallados a continuación y se presentan de forma sintetizada en la figura 1. En 1993 la recuperación de las finanzas públicas se asocia con un esfuerzo de contención del gasto y a un incremento de los ingresos fiscales debido a varios ajustes en las tarifas de los servicios públicos y a un incremento de los ingresos por impuestos por aduanas y ventas (Duran y Méndez, 1995), en 1994 la causa del gran déficit fiscal registrado se debe a la quiebra del Banco Anglo, por cuanto las pérdidas de este banco estatal fueron asumidas por el fisco. Posteriormente, en el 2002 el desmejoramiento de las finanzas públicas se le atribuye a un desequilibrio comercial (Fernández, 2005) y en el 2007, el mejoramiento de las finanzas públicas es producto de un incremento de la recaudación debido a que la economía mostró tasas de crecimiento del PIB de alrededor del $7 \%$ durante ese año. Por otra parte, en el año 2010 el déficit fiscal es un efecto del "Plan Escudo", el cual incluía incrementos de gastos por medio de subsidios, recapitalización de la banca e incrementos de la planilla del sector público, etc., aunado a una disminución de la carga fiscal en los años posteriores, debido a que la economía no recuperó su ritmo de crecimiento.

En promedio durante todo el periodo de estudio, de 22 años, se presenta un déficit 3,05\%, lo que muestra un claro sesgo deficitario en la política fiscal. Además, cabe resaltar que inmediatamente después de que se da una reducción considerable del déficit fiscal, y se alcanza un máximo en la serie, se producen periodos de altos déficits fiscales; este es el caso de los periodos 1993-1994 y 2007-2010. En otras palabras la reducción del déficit fiscal tarda al menos 10 años y una crisis fiscal se genera en uno o tres años. Esto ocurre debido a que el gasto público tiene un comportamiento meramente inercial y con una clara rigidez a la baja, por otra parte, el ingreso tributario se encuentra ligado mayormente al impuesto de ventas que depende principalmente del crecimiento económico.

Con lo que respecta a los niveles de endeudamiento público estos presentan un decrecimiento desde el año 1996 hasta el año 2008 donde alcanzó un 41 \% con respecto al PIB (figura 2). A partir del 2008 la deuda presenta una tendencia al alza marcada, principalmente, por el incremento del endeudamiento interno debido a la aplicación del 
"Plan Escudo" que provocó un gran déficit fiscal, el cual se mantuvo los años posteriores (2009-2013), debido al alto componente inercial del gasto público. Este incremento del endeudamiento interno provocó, en el 2012, presiones al alza en las tasas de interés del sistema financiero que su vez incentivaron el ingreso de capitales externos que buscaban aprovechar mayores rendimientos. Estas circunstancias ameritaron una intervención del poder ejecutivo que consistió en persuadir a los Bancos Estatales para que reduzcan sus niveles de tasas de interés, además de un cambio en la metodología de cálculo de la Tasa Básica Pasiva que excluye al Ministerio de Hacienda y al Banco Central $\stackrel{5}{\text {, }}$ y el canje de deuda interna por externa que consistió en la emisión de títulos de deuda externa por mil millones de dólares que viene a solventar las necesidades de financiamiento del Ministerio de Hacienda; de tal manera que reduce la presión causada por este ente en los mercados de deuda locales.

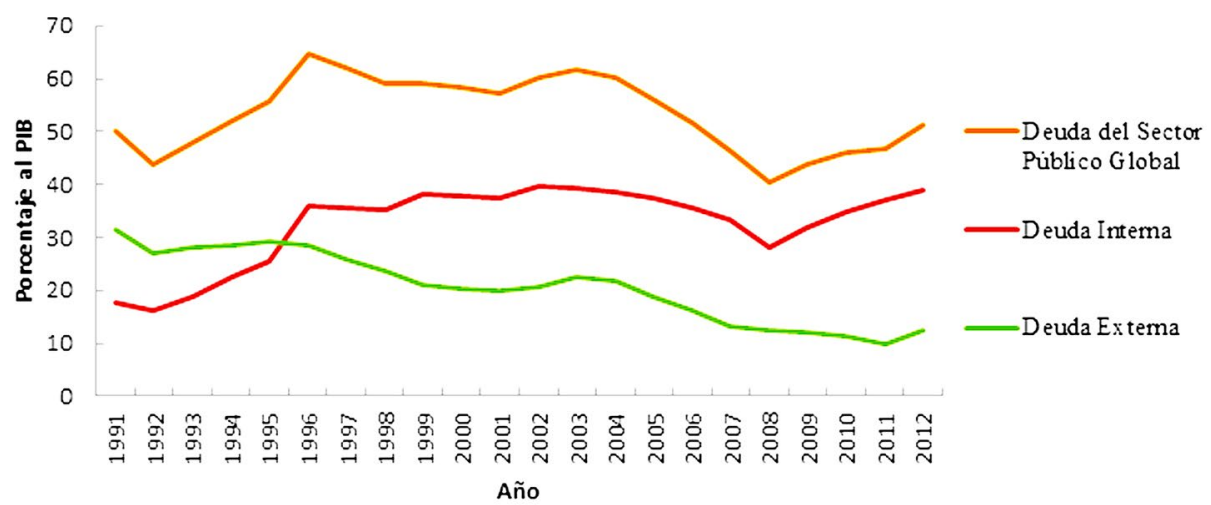

Figura 2. Deuda Total, Interna y Externa (1991-2012) -Porcentaje del Producto Interno Bruto- Fuente: elaboración propia con base en datos del Ministerio de Hacienda y la CEPAL

La economía costarricense ha presentado aversión al endeudamiento externo desde la crisis de la deuda externa en los 80 . Esto se refleja en la preferencia por la deuda interna desde la crisis de los ochentas y en una tendencia decreciente en la razón deuda externa PIB, tendencia que se revierte en el 2011 debido al canje de deuda.

El canje de deuda es una medida "paliativa", debido a que aprovecha la coyuntura internacional de bajas tasas de interés para reducir la presión sobre las tasas de interés locales y reducir el costo del servicio de la deuda. Sin embargo, de no existir un cambio estructural en el déficit fiscal es de esperarse que los niveles de deuda tanto interna como externa tengan una tendencia al alza, en dos sentidos: en primer lugar ante un déficit fiscal crónico la necesidad de financiamiento se mantiene y en segundo lugar el posible retiro de la política monetaria expansiva de Estados Unidos propiciaría incrementos en las tasas de interés externas que a su vez incrementan el pago del servicio de la deuda externa.

\footnotetext{
5 Además de excluir al Banco Central y el Ministerio de Hacienda la nueva metodología calcula la tasa como un promedio ponderado truncado y añade nuevas reglas de redondeo, de tal manera que evitan grandes variaciones en el nivel de la tasa, puesto que se convierte en un indicador más lento.
} 
Con respecto al futuro de la deuda y el déficit fiscal se identifican varios riesgos:

- El 42,7 \% del presupuesto 2014 es financiado con deuda; lo que indica que no se cubre el resultado financiero primario.

- El año 2014 es electoral lo que incrementa el gasto por deuda política.

- El 23 \% del presupuesto 2014 es destinado al pago del servicio de la deuda (figura 3).

- Se pronostica un bajo crecimiento económico, lo que disminuye recaudación tributaria.

- Se espera el retiro del estímulo monetario de la Reserva Federal de Estado Unidos de América, lo que compromete el endeudamiento externo y el canje de deuda debido a que dicho retiro incrementaría las tasas de interés internacionales.
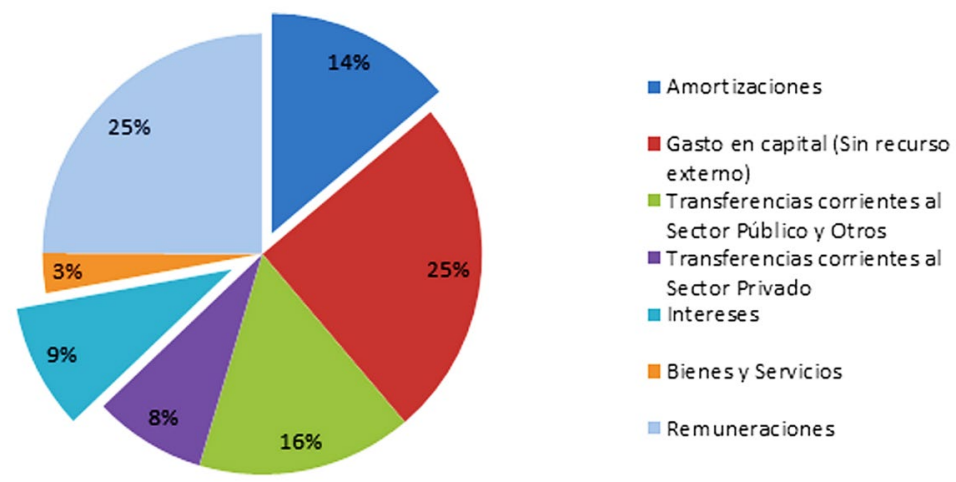

Figura 3. Presupuesto Nacional 2014 Fuente: elaboración propia con base en datos del Ministerio de Hacienda

\section{Resultados}

En esta sección se muestran los resultados obtenidos por la aplicación de la metodología. Se procederá inicialmente con el cálculo del límite crítico de deuda, luego con la estimación del modelo VAR y por último, se presentarán las simulaciones y el cálculo de las probabilidades que se desprende de estas.

La estimación del margen de deuda propuesto por Mendoza y Oviedo (2009) se estimó de la siguiente manera: el ingreso fiscal de peor escenario se estima en $\mathrm{t}^{\mathrm{min}}=9,2 \%$ al PIB; con una muestra de 92 observaciones (1991-2013) y con un nivel de confianza del $95 \%$.

De manera análoga, la estimación de la reducción del gasto de peor escenario resultó gin $=11 \%$ al PIB. La tasa de interés implícita promedio estimada es $r \approx 7,55 \%$, para el cálculo de las tasas se utilizó las tasas de interés de los bancos estatales a tres y seis meses ${ }^{6}$.

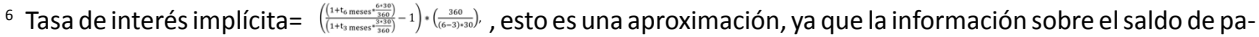
sivos totales y flujo de los costos por pasivos del Gobierno Central o Sector Público Global no es de dominio público.
} 
Por último el crecimiento promedio en estado estacionario es $\gamma=3,90 \%$; Se empleó como proxy la tendencia de Holdrick-Prescott, para lo que se usa el parámetro de suavizamiento propuesto por Segura \& Vásquez (2011).

Sustituyendo los valores obtenidos en la ecuación 5 , se obtiene el margen de deuda crítico $\left(d^{*}\right)$ :

$$
d^{*}=\frac{\left|t^{\min }-g^{\min }\right|}{|r-y|}=\frac{|0,0922742-0,112228|}{|0,0655-0,039031|}=54,71 \%
$$

Esto quiere decir que bajo el peor escenario de ingresos fiscales y en la mayor reducción posible del gasto, con un crecimiento en estado estacionario promedio y condiciones promedio de la tasa de interés, el límite de deuda critico a largo plazo es un $54,71 \%$ del PIB, por lo que los hacedores de política fiscal deben mantener la deuda bajo este margen para garantizar su sostenibilidad. Cabe resaltar que aunque, como muestra la figura 2, la deuda del sector público global ha superado este límite en años anteriores, esto no significa que la deuda sea insostenible en ese año, debido a que el límite critico depende de las condiciones del mercado (La tasa de interés, el crecimiento potencial y los ingresos y gastos del fisco) que son características de cada periodo de estudio ${ }^{7}$.

Por otra parte, el vector autorregresivo es estimado con 2 rezagos, lo que es resultado del test de rezago óptimo ${ }^{8}$ donde se utilizó el criterio de Schwarz. El orden de las variables del VAR representado por el vector $w_{t}$ (ecuación 8) es: crecimiento, déficit fiscal al PIB, tipo de cambio real, y por último tasa de interés. El orden de las variables permite una interpretación intuitiva de las ecuaciones ${ }^{9}$, aunque cabe resaltar que un modelo VAR es ateórico, es decir no es una representación estructural de la economía. La interpretación es propuesta por Tanner y Samake (2007) y se presenta a continuación:

- La primera ecuación del modelo VAR representa la oferta agregada y su variable dependiente es el crecimiento.

- La segunda es una ecuación fiscal y responde al comportamiento del gobierno y su variable dependiente es la razón déficit fiscal/PIB.

- La tercera ecuación es monetaria y representa el comportamiento del Banco Central ante efectos externos causados por la economía internacional, su variable es el tipo de cambio real.

- Por último, la cuarta ecuación es también de carácter monetario y representa el comportamiento local del Banco Central mediante una regla de Taylor, su variable es la tasa de interés.

\footnotetext{
7 Por ejemplo, aunque en 1996 la deuda alcanzó el 65 \% y el límite calculado con datos del 1991 al 2013 es un $54 \%$, la me-todología no indica que en 1996 la deuda fuera insostenible.

8 Véase anexo $\mathrm{B}$.

9 Las ecuaciones estimadas se encuentran en el anexo $\mathrm{E}$.
} 


\section{Simulaciones}

La simulación de las trayectorias de deuda se realiza utilizando los valores iniciales de 52,5 $\%$ de deuda/ PIB (valor observado en agosto del 2013), una tasa de interés inicial de 7,55\%, un déficit fiscal del 5,3\% al PIB y un crecimiento del 3,5\% (preliminar del BCCR para el 2013). Estos valores fueron actualizados con base en la revisión del programa macroeconómico del Banco Central en el tercer trimestre del 2013, las publicaciones de los indicadores económicos que realiza el Banco Central y en pronósticos realizados por los autores.

Estimación de la matriz M, propagadora de los choques en el sistema VAR:

$$
\left(\begin{array}{l}
\varepsilon_{1 t} \\
\varepsilon_{2 t} \\
\varepsilon_{3 t} \\
\varepsilon_{4 t}
\end{array}\right)=\left(\begin{array}{cccc}
0,0364 & 0,0000 & 0,0000 & 0,0000 \\
0,0031 & 0,0170 & 0,0000 & 0,0000 \\
-0,6460 & 0,6048 & 11,6490 & 0,0000 \\
-0,0147 & 0,0330 & 0,0366 & 0,2708
\end{array}\right)\left(\begin{array}{l}
v_{1 t} \\
v_{2 t} \\
v_{3 t} \\
v_{4 t}
\end{array}\right)
$$

Ya definidas las condiciones iniciales y estimada la matriz $M$ (ecuación 11), se realizó la simulación con una longitud temporal de tres años, es decir hasta el tercer trimestre del año 2016, y se repitió el experimento llevando a cabo 5000 iteraciones. Cada iteración significa una trayectoria generada de valores de deuda/PIB que es afectada por choques aleatorios distribuidos normalmente. Las 5000 trayectorias simuladas se presentan en la figura 4 y su promedio se representa con la línea negra.

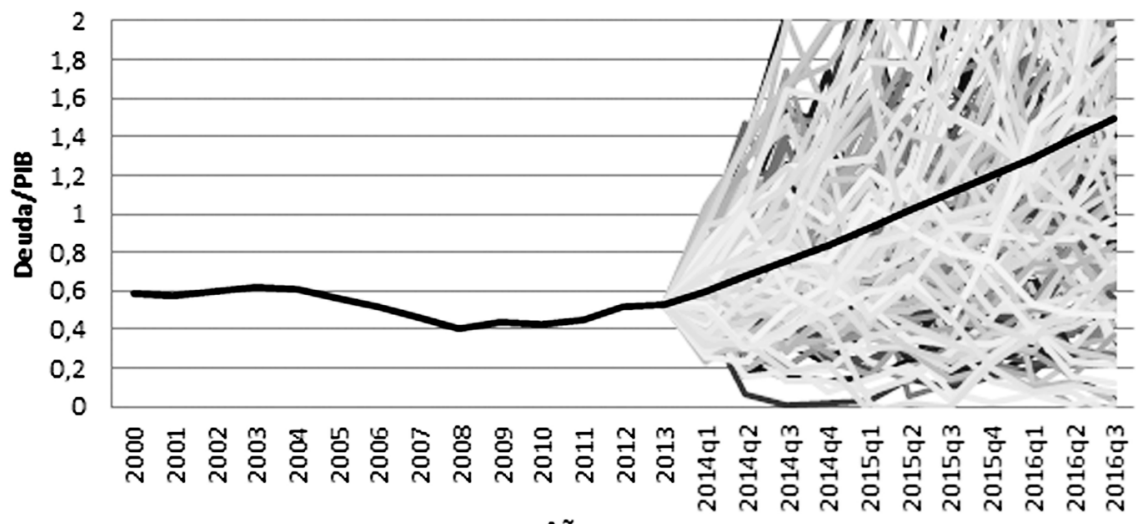

Año

Figura 4. Razón Deuda-PIB observada, y promedio de las simulaciones (2000-2016) Fuente: elaboración propia con base en las simulaciones realizadas

Para cada periodo se calcula la probabilidad de superar el límite de deuda de 54,71\%. Para validar estadísticamente las probabilidades estimadas, es importante saber que estos valores convergen o divergen a un valor estable ${ }^{10}$ y único. El experimento refleja que todas las probabilidades convergen ya en un número de iteraciones cercano a 1500 (figura 5),

\footnotetext{
10 Estadísticamente se hace alusión en este caso al concepto de convergencia en el sentido de Cèsaro, veáse Dhrymes (1970) pág 389. Por otra parte, la Real Academia Española define estable como: 1. adj. Que se mantiene sin peligro de cambiar, caer o desaparecer. 2. adj. Que permanece en un lugar durante mucho tiempo. 3. adj. Que mantiene o recupera el equilibrio.
} 
lo cual indica que los valores de probabilidad son únicos y estables. Se realizan 5000 iteraciones para mayor precisión, teóricamente cuando el número de iteraciones tiende a infinito los valores de probabilidad se acercan a sus valores "reales" (Dhrymes, 1970).

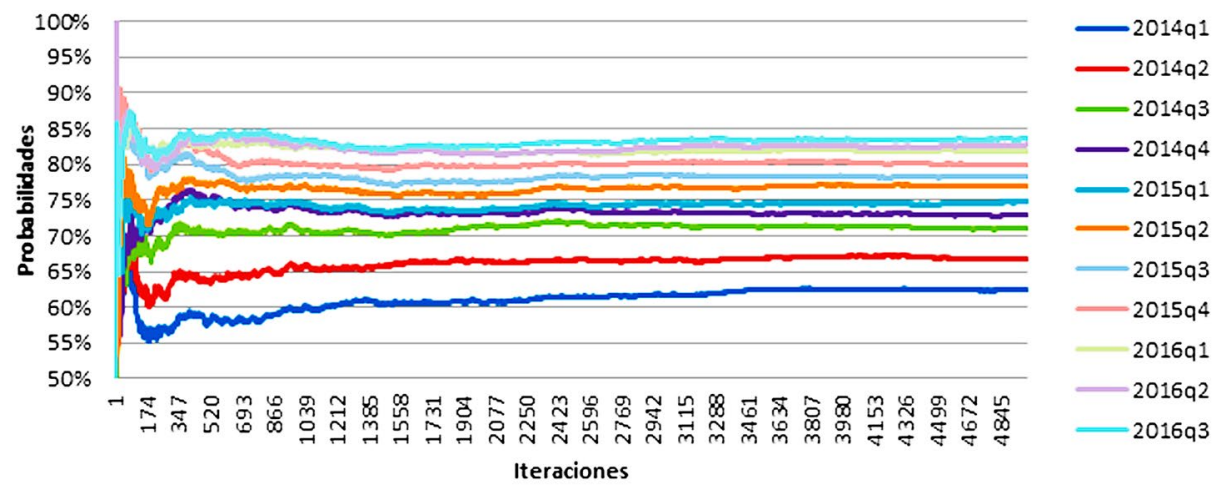

Figura 5. Convergencia de probabilidad -5 000 Iteraciones- Fuente: elaboración propia con base en las simulaciones realizadas

Los valores estimados de probabilidad se presentan en la figura 6 y los valores indican que el país tiene, al tercer trimestre del 2014, una probabilidad de un 50,20\% de superar el límite de deuda crítico, al tercer trimestre del 2015 un 76,81 \% y para el 2016 se calcula en $82,22 \%$. Esto representa un riesgo para el país dado que probabilidades mayores a $50 \%$ son señal de alerta. En promedio para el periodo 2014q4-2016q3 la probabilidad se estima en un $76,09 \%$.

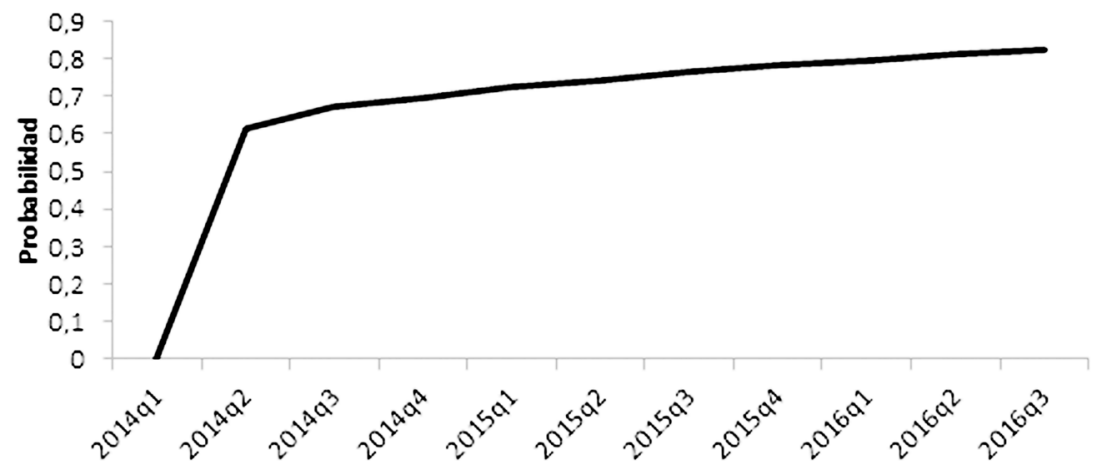

Figura 6. Probabilidad de superar el margen crítico de deuda Fuente: elaboración propia con base en las simulaciones realizadas.

Un insumo adicional resultante de la estimación del VAR, utilizado para estimar la descomposición de Cholesky del modelo, es la posibilidad de realizar simulaciones de política económica mediante funciones de impulso-respuesta. 
El análisis de las funciones de impulso-respuesta del Vector Autorregresivo muestra que un aumento de la tasa de interés de 1 punto porcentual ( 1 p.p. $)^{11}$ incrementa el déficit en 0,11 p.p., por lo que mantener la tasa de interés de la economía en niveles bajos propiciaría la sostenibilidad de la deuda. Por otra parte, una depreciación del tipo de cambio real de 1 p.p. tendría un pequeño impacto en la reducción del déficit en un 0,043 p.p., este efecto se revertiría al menos en dos años y el déficit tendería a aumentar un pequeño porcentaje, por lo que la política cambiaria no tiene un efecto significativo sobre la sostenibilidad de la deuda a largo plazo, y aquí se consideran las variables en niveles; pero cabe resaltar que en un contexto cambiario inestable el riesgo de las variaciones cambiarias incrementa el servicio de deuda externa. Por lo que es necesaria cierta estabilidad relativa del tipo de cambio para garantizar la sostenibilidad de la deuda.

El mayor impacto sobre el stock de deuda se alcanza mediante variaciones del crecimiento económico,cuandolaeconomíacrecelacargafiscalaumentaylanecesidaddefinanciamiento del déficit disminuye, por lo tanto también la deuda pública; como indica la ecuación 3.

Las estimaciones de las funciones de impulso-respuesta reflejan que una reducción del déficit fiscal, causado por una política fiscal contractiva (incremento de ingresos o reducción de gastos), tiene un efecto negativo a corto plazo sobre el crecimiento debido a la contracción de la demanda agregada, pero la dinámica de ajuste de la variable crecimiento ante esta política indica que a largo plazo existe un efecto positivo sobre el crecimiento. La reducción de la proporción del déficit al PIB de 1p.p. provocaría una reducción de la tasa de crecimiento de un 0,36 p.p. a corto plazo y un aumento de la tasa de crecimiento de 0,1764p.p. a largo plazo (efecto acumulado), lo que, por las razones antes indicadas, tiene un efecto positivo en términos de la sostenibilidad de la deuda, ya que esta combinación de factores propiciaría que el stock de deuda se mantenga en un nivel menor al nivel crítico.

En resumen, una reducción del déficit fiscal es prioritaria para disminuir la cantidad de endeudamiento públicoyasegurar la sostenibilidad fiscal. Además, la reducción del déficites vital para aminorar la presión sobre las tasas de interés causado por las captaciones del fisco en los mercados internos de deuda. Adicionalmente, la reducción del stock de deuda y del déficit fiscal es fundamental para no comprometer el crecimiento económico a largo plazo.

\section{Análisis de sensibilidad de los resultados}

Para determinar la sensibilidad de los valores de probabilidad estimados ante cambios en sus condiciones iniciales, se realizó un análisis de sensibilidad. Este consistió en cuantificar la desviación promedio entre los valores de probabilidad de la simulación base y los valores resultantes de simulaciones con alteraciones de un 1p.p. en sus condiciones

11 El impulso-respuesta se presenta normalizado a 1 para facilitar al lector su interpretación, las funciones estimadas, en su escala original se encuentran en el anexo $C$ y $D$. 
iniciales. La prueba demostró que los valores de probabilidad se mantienen estables y sin modificaciones considerables si las condiciones iniciales del modelo son cambiadas ${ }^{12}$.

\section{Consideraciones finales}

A través de la aplicación de la metodología estocástica de evaluación de la sostenibilidad fiscal se concluye lo siguiente:

A. Basado en los resultados se puede observar que Costa Rica, actualmente, se encuentra en un escenario de riesgo para la sostenibilidad fiscal, debido a que las estimaciones de las probabilidades indican que el país tiene en promedio, para el periodo 2014q4-2016q3, un 76,09\% de probabilidad de superar el límite crítico y probabilidades mayores $50 \%$, las cuales son señal de alerta.

B. A través del análisis de las funciones de impulso-respuesta se estima que una reducción de la proporción del déficit al PIB de 1 p.p. provocaría un aumento de la tasa de crecimiento en el largo plazo de 0,1764p.p., lo que favorece la reducción del stock de deuda y garantiza la sostenibilidad de la misma.

C. Basado en los resultados obtenidos en simulaciones se propone como recomendación general aumentar el ingreso del gobierno mediante una reforma fiscal y/o una reducción de gastos, esto con el fin de evitar el incremento de la deuda y una posterior presión al alza de las tasas de interés locales y de la tasa de a inflación. De esta forma se revertiría la actual insostenibilidad de la deuda.

D. Es importante considerar no solo una reforma, sino una migración hacia sistemas de política óptima o de reglas de política fiscal. Esta forma de ejecutar la política fiscal parece ser la forma de eliminar el sesgo deficitario existente y lograr una política fiscal estabilizadora que asegure la sostenibilidad de la deuda pública.

Para cuantificar la magnitud de esta reducción del gasto o incremento de impuestos necesarios para revertir la tendencia creciente de la deuda pública los autores actualmente trabajan en un modelo macroeconómico de pequeña escala. Este es un modelo de ecuaciones simultáneas especificado desde la síntesis de la nueva economía Keynesiana (microfundamentado) que permite realizar las simulaciones correspondientes mediante distintos escenarios.

12 Véase Anexo F. 


\section{Referencias}

Acevedo, C. (2001). ¿Es sostenible la política fiscal en el Salvador? FUSADES. Recuperado de: http://www.fusades.org/index.php?option=com idownloads\&/temid=95\&view =finish\&cid=213\&catid=36\&lang=es

Blanchard, O. (1990). Suggestions for a new set of fiscal indicators. OECD working paper $\mathrm{n}$. 79. Recuperado de: http://www.oecd.org/tax/public-finance/2002735.pdf

Buiter, W. (November, 1985). A Guide to Public Sector Debt and Deficits. Economic Policy, 21. doi: http://dx.doi.org/10.2307/1344612

De Gregorio, J. (2007). Macroeconomía: Teoría y políticas. México: Pearson Education. Recuperado de: http://www.degregorio.cl/

Dhrymes, P. (1970). Econometrics. Statistical foundations and aplications. Berlin: SpringerVerlag. Recuperado de: http://www.springer.com/statistics/book/978-0-38790095-7

Fernández, K. (2005). Evaluación de la Sostenibilidad Fiscal en Costa Rica: Un Enfoque Estocástico. (Tesis de maestría). Pontificia Universidad Católica de Chile, Chile. Recuperado de: http://www.economia.puc.cl/docs/tesis kfernandez.pdf

Ghysels, E. (1990). Unit-Root Tests and the Statistical Pitfalls of Seasonal Adjustment: The Case of U.S. PostwarReal Gross National Product. Journal of Business \& Economic Statistics, 8(2),145-152.doi:http://dx.doi.org/10.1080/07350015.1990.10509785

Ghysels, E., \& Perron , P. (1993). The Effect of Seasonal Adjustment Filters on Tests for a Unit Root. Journal of Econometrics, 55(1-2), 57-98. doi: http://dx.doi. org/10.1016/0304-4076(93)90004-0

Hamilton, J. (1994). Time series analysis. United States of America: Princeton University. Recuperado de: http://press.princeton.edu/titles/5386.html

Hansen, L. P., \& Sargent, T. J. (January-February, 1993). Seasonality and aproximation errors in rational expectation models. Journal of Econometrics, 55(1-2), 21-55. doi: http://dx.doi.org/10.1016/0304-4076/93)90003-N

Keynes, J. M. (1971). Breve tratado sobre la reforma monetaria. Mexico: Fondo de Cultura Económica. Recuperado de: http://www.fondodeculturaeconomica.com/ librerias/Detalle.aspx?ctit $=001604 R$

Méndez, E., \& Durán, R. (1995). Orientación discrecional y cíclica de la política fiscal en Costa Rica. Recuperado de: http://www.bccr.fi.cr/investigacioneseconomicas/ sectorpublico/Orientacion Discrecional CiclicPolitica Fiscal en Costa Rica.pdf 
Mendoza, E., \& Oviedo, M. (July, 2004). Public debt, fiscal solvency and macroeconomic uncertainty in Latin America: The cases of Brazil, Colombia, Costa Ricam and Mexico. Recuperado de: http://www.nber.org/papers/w10637

Papadopoulos, A. P. y Sidiropoulos, M. G. (August, 1999). The Sustainability of Fiscal Policies in the European Union. International Advances in Economic Research, 5(3). doi: http://dx.doi.org/10.1007/BF02296413

Ross, S. (1999). Simulación. Mexico: Prentice Hall.

Segura, C., \& Vásquez, J. P. (2011). Estimación del parámetro de suavizamiento del filtro de Hodrick y Prescott para Costa Rica. Recuperado de: http://www.bccr.fi.cr/ investigacioneseconomicas/metodoscuantitativos/Estimacion del parametro de suavizamiento del filtro de Hodrick $y$ Prescott para CR.pdf

Tanner, E., \& Samake., I. (2007). Sostenibilidad probabilística de la deuda pública: Un enfoque de vectores autorregresivos para los casos de Brasil, México y Turquía. Recuperado de: http://www.captac-dr.org/c/document library/get file?folderld=107574\&name=DLFE-4858.pdf

Talvi, E. \& Végh, C., (1998). Fiscal Policy Sustainability: A Basic Framework. Recuperado de: http://idbdocs.iadb.org/wsdocs/getdocument.aspx?docnum=787999

\section{Anexos}

\section{A. Código de Matlab.}

\%\% Sostenibilidad estocástica de la deuda de Costa Rica, $\quad \% \% \%$

\%\%\% Una aproximación mediante el Método Montecarlo. UNA (2012). \%\%\%\%

\%NOTAS:

\% Esta es una versión modificada del modelo de K Fernández (2005).

$\%$ Con el objetivo de calcular la probabilidad

$\%$ de sobre pasar el límite de deuda a largo plazo propuesto por Mendoza y Oviedo (2007), $\%$ a través del Método Montecarlo.

\% Se utiliza el procedimiento de simulación de Tanner y Samake (2007), FMI.

function fsVAR2

close all

clc

tic

$\mathrm{n}=\mathrm{xxx}$;

$\mathrm{T}=\mathrm{xxx}$;

\%Número de iteraciones

$\mathrm{D} 0=\mathrm{xxx}$;

\%Horizonte temporal

Dstar $=\mathbf{x x x}$;

\%valor inicial de la deuda 
mean $\mathrm{R}=\mathrm{xxx} ;$
mean $=\mathrm{xxx} ;$
meanC $=x x x ;$
meanE $=x x x ;$
Cont $=0 ;$

$\mathrm{stdE}=\mathrm{xxx}$;

\%\%\%\% Matriz de shocks \%\%\%\%\%\%\%\%\%\%\%\%\%\%\%\%\%\%

Covh $=$ xlsread('XXXXXXXXXXX.XIs');

Cho=chol(Covh,'lower');

$\% \% \% \% \% \% \%$ Simulación de trayectorias \%\%\%\%\%\%\%\%\%\%\%\%

$\mathrm{H}=$ waitbar(0,'Calculando...');

for $i=1: 1:(n)$

$\mathrm{D}(1, \mathrm{i})=\mathrm{D0}$;

waitbar(i/n)

for $\mathrm{t}=2: 1: \mathrm{T}$

Ep=Cho*randn $(4,1)$;

$\mathrm{E}(\mathrm{t}, \mathrm{i})=$ mean $\mathrm{E}+\mathrm{std} \mathrm{E}^{*}$ randn;

$\mathrm{C}(\mathrm{t}, \mathrm{i})=$ mean $\mathrm{C}+\mathrm{Ep}(1,1)$;

$\mathrm{R}(\mathrm{t}, \mathrm{i})=$ mean $\mathrm{R}+\mathrm{Ep}(4,1)$;

$\mathrm{G}(\mathrm{t}, \mathrm{i})=$ mean $\mathrm{G}+\mathrm{Ep}(2,1)$;

$D(t, i)=(1+R(t, i)-C(t, i)) *(D(t-1, i))-G(t, i)+E(t, i)$;

end

end

\%\%\%\%\%\%\%\%\%\%\%\%\%\% Contador lógico \%\%\%\%\%\%\%\%\%\%\%\%\%\%\%\%\%\%\%\%

for $\mathrm{t}=2: 1: \mathrm{T}$

for $\mathrm{i}=2: 1:(\mathrm{n})$;

Cont $(t, 1)=0$;

if $D(t, i)>$ Dstar

$\operatorname{Cont}(t, i)=\operatorname{Cont}(t, i-1)+1$;

else

$\operatorname{Cont}(\mathrm{t}, \mathrm{i})=\operatorname{Cont}(\mathrm{t}, \mathrm{i}-\mathrm{-})$;

end

$P(t, i)=\operatorname{Cont}(t, i) /(i-1)$;

end

$\operatorname{Ser}(t, 1)=P(t, n)$;

end

close $(\mathrm{H})$

\%\%\%\%\%\%\%\%\%\%\%\% Graficando \%\%\%\%\%\%\%\%\%\%\%\%\%\%\%\%\%\%\%\%\%\%

hold on

plot(D)

axis([1 T -1 4])

title('Trayectorias Simuladas de deuda')

xlabel('Periodo $t^{\prime}$ )

ylabel('Deuda/Pib') 


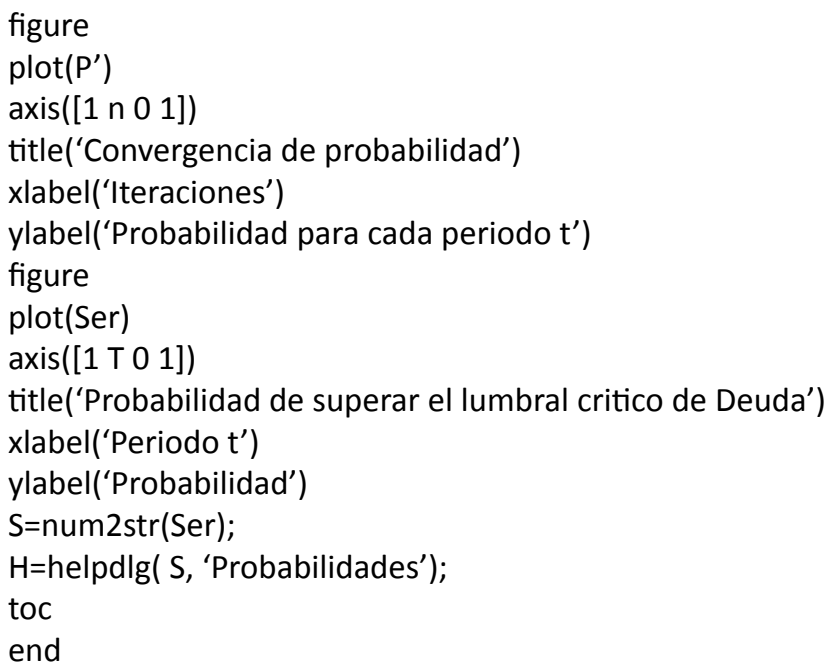

\%\%\%\%\%\%\%\%\%\%\%\%\%\%\%\%\%\%\%\%\%\%\%\%\%\%\%\%\%\%\%\%\%\%\%\%\%\%\%\%\%\%\%\%\%\%\%\%\% $\%$ Autor del código:

\%Bach. Marco Vinicio Valerio Berrocal.

\%Estudiante de Economía de la Universidad Nacional de Costa Rica.

\%Consultas: markxsw@gmail.com

\%\%\%\%\%\%\%\%\%\%\%\%\%\%\%\%\%\%\%\%\%\%\%\%\%\%\%\%\%\%\%\%\%\%\%\%\%\%\%\%\%\%\%\%\%\%\%\%\%

\section{B. Elección del rezago óptimo Vector autorregresivo}

\begin{tabular}{|c|c|c|c|c|c|c|}
\hline \multicolumn{7}{|c|}{ Criterios de selección identificar el rezago óptimo } \\
\hline Rezago & $\begin{array}{l}\text { Logaritmo de } \\
\text { verosimilitud }\end{array}$ & $\begin{array}{c}\text { Razón de } \\
\text { Quasi- } \\
\text { verosimilitud }\end{array}$ & $\begin{array}{c}\text { Error final de } \\
\text { predicción }\end{array}$ & Akaike & Schwarz & $\begin{array}{c}\text { Hannan } \\
\text { Quin }\end{array}$ \\
\hline 0 & 444,60 & NA & $7.56 e-12$ & $-11,42$ & $-11,27$ & $-11,36$ \\
\hline 1 & 722,29 & 512,10 & $1.07 e-14$ & $-17,98$ & $-17,07$ & $-17,16$ \\
\hline$\underline{\mathbf{2}}$ & 790,87 & 117,57 & $3.48 e-15$ & $-19,11$ & $-\underline{17.43^{*}}$ & $-18.44^{*}$ \\
\hline 3 & 818,96 & 44,50 & $3.27 e-15$ & $-19,19$ & $-16,76$ & $-18,22$ \\
\hline 4 & 848,70 & $43,26 *$ & $3.01 \mathrm{e}-15^{*}$ & $-19.31 *$ & $-16,12$ & $-18,04$ \\
\hline 5 & 866,07 & 23,00 & $3.93 e-15$ & $-19,12$ & $-15,16$ & $-17,54$ \\
\hline 6 & 891,33 & 30,18 & $4.33 e-15$ & $-19,13$ & $-14,41$ & $-17,24$ \\
\hline 7 & 910,38 & 20,29 & $5.88 e-15$ & $-18,97$ & $-13,49$ & $-16,78$ \\
\hline
\end{tabular}


C. Funciones de Impulso- Repuesta (IRF) del modelo VAR (Gráficos Combinados).

Respuesta del crecimiento ante una perturbacion de un desvio estandar(Cholesky)

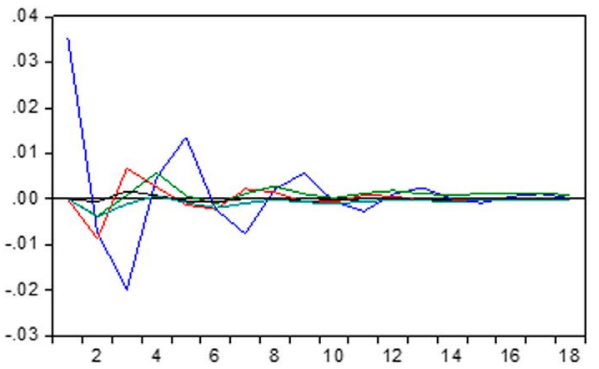

Respuesta del tipo de cambio real ante una perturbacion de un desvio estandar(Cholesky)

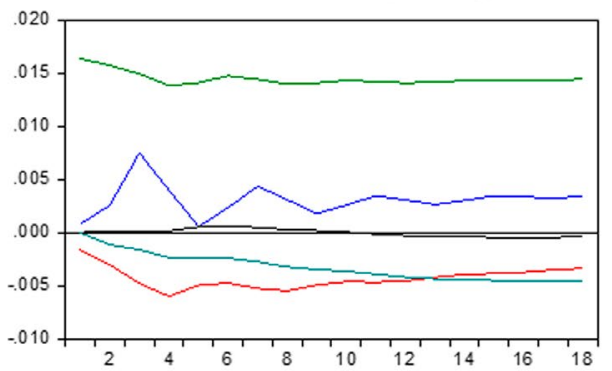

Respuesta de la razon deficitPIB ante una perturbacion de un desvio estandar(Cholesky)

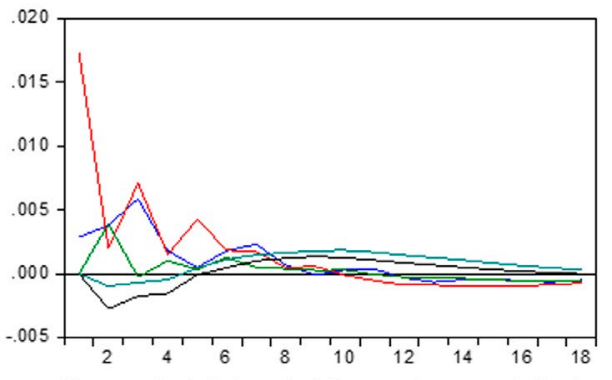

Respuestade la tasa de interes ante una perturbacion de un desvio estandar(Cholesky)

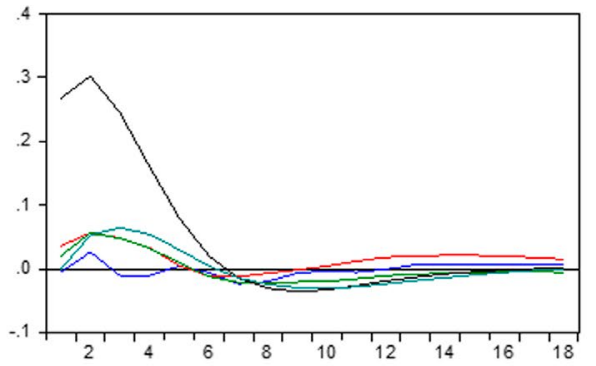

$$
\begin{array}{|l|}
\hline \text { Crecimiento } \\
\text { De ficit/PIB } \\
\text { - Tipo de cambio real } \\
\hline \text { T asa de interes } \\
\text { Deuda }
\end{array}
$$

Julio César Espinosa Rodríguez, Marco Vinicio Valerio Berrocal

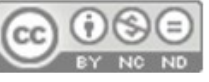



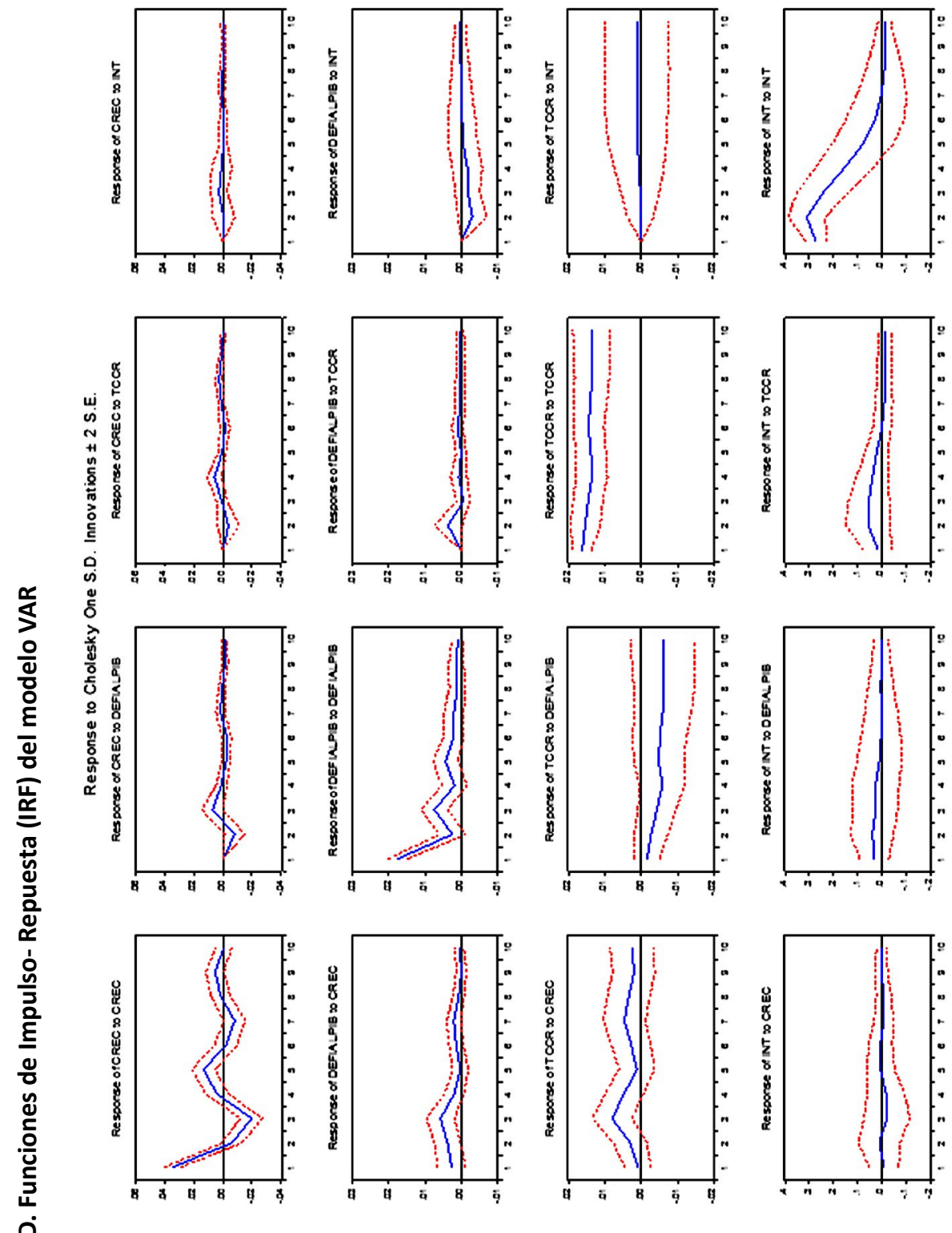


\section{E. Estimación del modelo VAR}

Vector Autoregression Estimates

Included observations: 82 after adjustments

Standard errors in () \& t-statistics in [ ]

\begin{tabular}{|c|c|c|c|c|}
\hline & CREC & DEFIALPIB & TCCR & INT \\
\hline \multirow[t]{3}{*}{$\operatorname{CREC}(-1)$} & -0.128579 & 0.083944 & 0.065587 & 0.505016 \\
\hline & $(0.09220)$ & $(0.04610)$ & $(0.04288)$ & $(0.72181)$ \\
\hline & {$[-1.39455]$} & [ 1.82072] & [ 1.52961] & [ 0.69965] \\
\hline \multirow[t]{3}{*}{$\operatorname{CREC}(-2)$} & -0.552361 & 0.114846 & 0.168685 & -1.163416 \\
\hline & $(0.08787)$ & $(0.04394)$ & $(0.04086)$ & $(0.68792)$ \\
\hline & {$[-6.28604]$} & [ 2.61372$]$ & [ 4.12789] & {$[-1.69122]$} \\
\hline \multirow[t]{3}{*}{ DEFIALPIB $(-1)$} & -0.486134 & 0.179662 & -0.074960 & 0.499990 \\
\hline & $(0.19693)$ & $(0.09847)$ & $(0.09158)$ & (1.54169) \\
\hline & {$[-2.46858]$} & [ 1.82447$]$ & {$[-0.81850]$} & [0.32431] \\
\hline \multirow[t]{3}{*}{ DEFIALPIB(-2) } & 0.418476 & 0.464173 & -0.053687 & -0.128009 \\
\hline & $(0.20082)$ & $(0.10042)$ & (0.09339) & (1.57219) \\
\hline & [ 2.08380$]$ & {$[4.62226]$} & {$[-0.57485]$} & {$[-0.08142]$} \\
\hline \multirow[t]{3}{*}{$\operatorname{TCCR}(-1)$} & -0.226284 & 0.234080 & 0.952554 & 2.389205 \\
\hline & $(0.22805)$ & $(0.11404)$ & $(0.10605)$ & (1.78533) \\
\hline & {$[-0.99226]$} & [ 2.05270$]$ & [ 8.98172] & [ 1.33824$]$ \\
\hline \multirow[t]{3}{*}{$\operatorname{TCCR}(-2)$} & 0.347327 & -0.249499 & 0.025854 & -2.541822 \\
\hline & $(0.22979)$ & $(0.11490)$ & $(0.10686)$ & (1.79893) \\
\hline & [1.51152] & {$[-2.17136]$} & {$[0.24194]$} & {$[-1.41296]$} \\
\hline \multirow[t]{3}{*}{ INT(-1) } & -0.001490 & -0.011243 & 0.000672 & 1.138022 \\
\hline & $(0.01428)$ & $(0.00714)$ & $(0.00664)$ & $(0.11178)$ \\
\hline & {$[-0.10437]$} & {$[-1.57471]$} & {$[0.10116]$} & [ 10.1814$]$ \\
\hline \multirow[t]{3}{*}{ INT(-2) } & 0.006224 & 0.008225 & -0.001047 & -0.396736 \\
\hline & $(0.01433)$ & $(0.00717)$ & $(0.00667)$ & $(0.11220)$ \\
\hline & {$[0.43426]$} & [1.14767] & {$[-0.15707]$} & {$[-3.53586]$} \\
\hline \multirow[t]{3}{*}{ c } & .0 .095611 & 0.022729 & 0.006145 & 2.197115 \\
\hline & $(0.07582)$ & $(0.03791)$ & $(0.03526)$ & $(0.59358)$ \\
\hline & {$[-1.26101]$} & [0.59948] & {$[0.17428]$} & [ 3.70148$]$ \\
\hline R-squared & 0.467843 & 0.367353 & 0.987481 & 0.694919 \\
\hline Adj. R-squared & 0.409524 & 0.298022 & 0.986109 & 0.661486 \\
\hline Sum sq. resids & 0.089843 & 0.022465 & 0.019431 & 5.506318 \\
\hline S.E. equation & 0.035082 & 0.017542 & 0.016315 & 0.274643 \\
\hline F-statistic & 8.022192 & 5.298534 & 719.7456 & 20.78510 \\
\hline Log likelihood & 163.1201 & 219.9504 & 225.8998 & -5.619210 \\
\hline Schwarz SC & -3.494874 & -4.880981 & -5.026087 & 0.620718 \\
\hline Mean dependent & 0.041577 & -0.026225 & 1.029187 & 7.653730 \\
\hline S.D. dependent & 0.045654 & 0.020938 & 0.138423 & 0.472042 \\
\hline \multicolumn{2}{|c|}{ Determinant resid covariance (dof adj.) } & $7.22 \mathrm{E}-12$ & & \\
\hline \multicolumn{2}{|c|}{ Determinant resid covariance } & $4.53 \mathrm{E}-12$ & & \\
\hline \multicolumn{2}{|c|}{ Log likelihood } & 605.4940 & & \\
\hline \multicolumn{2}{|c|}{ Akaike information criterion } & -13.89010 & & \\
\hline \multicolumn{2}{|c|}{ Schwarz criterion } & -12.83349 & & \\
\hline
\end{tabular}

Julio César Espinosa Rodríguez, Marco Vinicio Valerio Berrocal

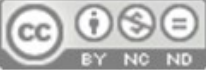




\section{F. Análisis de sensibilidad del modelo.}

\begin{tabular}{|c|c|c|c|c|c|}
\hline & \multicolumn{5}{|c|}{ Análisis de Sensibilidad } \\
\hline & \multirow{2}{*}{$\begin{array}{c}\text { Probabilidad } \\
\text { Estimada }\end{array}$} & \multicolumn{4}{|c|}{$\begin{array}{l}\text { Desviación en puntos porcentuales (p.p.) del valor de probabilidad ante una Variación } \\
\text { en } 1 \text { p.p. de las condiciones iniciales del modelo }\end{array}$} \\
\hline & & Crecimiento & Déficit & Tasa de Interés & Deuda \\
\hline \multirow{2}{*}{\begin{tabular}{l|l}
1 \\
2
\end{tabular}} & $61,1 \%$ & $-0,01$ & $-0,02$ & $-0,01$ & 0,05 \\
\hline & $67,4 \%$ & 0,02 & $-0,03$ & 0,01 & 0,04 \\
\hline \multirow{2}{*}{$\begin{array}{l}3 \\
4\end{array}$} & $69,4 \%$ & 0,02 & $-0,06$ & 0,02 & 0,03 \\
\hline & $72,4 \%$ & 0,02 & $-0,06$ & 0,03 & 0,02 \\
\hline \multirow{2}{*}{$\begin{array}{l}5 \\
6\end{array}$} & $74,2 \%$ & 0,02 & $-0,07$ & 0,04 & 0,02 \\
\hline & $76,5 \%$ & 0,03 & $-0,08$ & 0,03 & 0,02 \\
\hline \multirow{2}{*}{$\begin{array}{l}7 \\
8\end{array}$} & $78,3 \%$ & 0,03 & $-0,07$ & 0,03 & 0,02 \\
\hline & $79,3 \%$ & 0,03 & $-0,06$ & 0,02 & 0,03 \\
\hline \multirow{2}{*}{$\begin{array}{c}9 \\
10 \\
\end{array}$} & $81,2 \%$ & 0,03 & $-0,06$ & 0,02 & 0,02 \\
\hline & $82,6 \%$ & 0,03 & $-0,05$ & 0,01 & 0,02 \\
\hline & & \multicolumn{4}{|c|}{ Desviación Promedio } \\
\hline & & 0,02 & $-0,06$ & 0,02 & 0,03 \\
\hline
\end{tabular}

NOTA: No son escenarios de simulación. 